\title{
Estimation of habitual dietary heterocyclic amine intake in a UK population
}

\author{
S.F. Brennan ${ }^{1}$, M. Cantwell ${ }^{2}$, J.V. Woodside ${ }^{2}$, K. Cooper ${ }^{1}$ and G.J. Cuskelly ${ }^{1}$ \\ ${ }^{1}$ Institute for Global Food Security, Malone Road, Queen's University Belfast,BT9 $5 B N$ and ${ }^{2}$ Centre for Public Health, \\ Grosvenor Road, Queen's University Belfast, BT12 6BA
}

High meat intake has been linked to an increased incidence of some cancers in epidemiological studies ${ }^{(1,2)}$. Compounds formed during cooking of meat, such as heterocyclic amines (HCAs), are thought to influence cancer risk. As the extent of heterocyclic amine formation is dependent upon cooking method, temperature of cooking and cooking time, accurate estimation of exposure is difficult and limited data are available. In this study we aim to estimate habitual dietary heterocyclic amine intake from detailed 7-day food diaries for the first time in a UK population.

Healthy participants $(n=155)$ were recruited and those under 18 years and smokers were excluded (due to exposure to polycyclic aromatic hydrocarbons). Participants completed a 7-day food diary and received detailed instructions on how to record their dietary intake, with particular emphasis on describing cooking methods and 'doneness' level of all meat consumed. Food diaries were reviewed after completion and participants were asked to confirm/clarify the food portions they recorded in their food diary using a photographic portion size atlas. Participants were asked to clarify the cooking methods used and to describe the 'doneness' of any meat consumed by selecting from a series of published colour-printed 'meat -doneness' pictures. Dietary HCA values (including individual HCAs; MeIQx, DiMeIQx and PhIP) were included in nutritional analyses by updating the dietary analysis program, WISP v3.0 (Tinuviel, UK), with additional meat codes based on cooking methods/ 'doneness' level. Meat content values for total and individual HCAs were assigned to the newly updated food codes and cooking methods. Information on HCA content was obtained primarily from the CHARRED online database. HCA values were applied directly to 'meat only' food codes and to composite dishes (calculated from disaggregated meat content). Disaggregated meat content was calculated using published data or estimated from average percentage meat content in commercial dishes available from UK supermarkets.

\begin{tabular}{|c|c|c|c|c|}
\hline & Tertile $1(\mathrm{n}=51)$ & Tertile $2(n=52)$ & Tertile $3(n=53)$ & $\mathrm{P}$ \\
\hline Total HCA (ng/d) & $85.9(65.7)$ & $420.7(141.6)$ & $1090 \cdot 3(417 \cdot 0)$ & \\
\hline MeIQx (ng/d) & $27.3(28.9)^{\mathrm{a}}$ & $94.6(57.3)^{\mathrm{b}}$ & $183.1(104.8)^{\mathrm{c}}$ & $<0.01$ \\
\hline DiMeIQx (ng/d) & $10 \cdot 0(18.8)^{\mathrm{a}}$ & $19 \cdot 4(20 \cdot 3)^{\mathrm{a}}$ & $38.5(33.0)^{\mathrm{b}}$ & $<0.01$ \\
\hline $\mathrm{PhIP}$ (ng/d) & $48.6(49 \cdot 5)^{\mathrm{a}}$ & $306.7(121.0)^{\mathrm{b}}$ & $871.9(333.9)^{\mathrm{c}}$ & $<0.01$ \\
\hline Energy (kcal/d) & $1649 \cdot 6(543 \cdot 1)$ & $1709.6(597.8)$ & $1881.9(537.0)$ & 0.09 \\
\hline Total fat $(\mathrm{g})$ & $62.9(22.2)$ & $67.6(25.4)$ & $74 \cdot 0(24 \cdot 3)$ & 0.06 \\
\hline Saturated fat $(\mathrm{g})$ & $21.7(9.6)$ & $23.8(10 \cdot 9)$ & $26 \cdot 3(9.7)$ & 0.07 \\
\hline
\end{tabular}

Data shown are mean (SD). Differences between tertiles were analysed using by one-way ANOVA (significant at $\mathrm{p}<0 \cdot 05$ ). Means within a row with different superscript letters are significantly different (Tukey-B post hoc)

Daily intakes of total HCA were calculated by tertile. MeIQx, DiMeIQx and PhIP (ng/d) were reported per tertile and energy intake $(\mathrm{kcal} / \mathrm{d})$ and total and saturated fat $(\mathrm{g} / \mathrm{d})$ were calculated by tertile of total heterocyclic intake (see table).

Habitual levels of HCA intake were estimated for the first time in a UK population via collection of detailed 7-day food diary data. Energy intake, total and saturated fat intake tended to increase with increasing HCA intake but results were not significant. It is of interest to develop a UK-specific database of HCA content of foods to further improve accuracy of dietary HCA estimation.

1. Sinha R (2002) Mutat. Res-Fund. Mol. M. 506, 197-204.

2. Kim E, Coelho D, Blachier F (2013) Nutr Res, 33(12): 983-94. 